\title{
Impact of Initial Thrombocytopenia on Short and Midterm Outcomes in Patients with Acute Coronary Syndromes Who Underwent Percutaneous Coronary Intervention
}

\author{
SHERIF W. AYAD, M.D.; MOHAMED A. SADAKA, M.D.; MOHAMED A. SOBHY, M.D. and \\ OLA S. NOUR ELDIN, M.Sc. \\ The Department of Cardiology, Faculty of Medicine, Alexandria University, Egypt
}

\begin{abstract}
Background: Acute Coronary Syndromes (ACS) patients with thrombocytopenia are challenging in their management as they carry high bleeding and thrombotic risks.

Aim of Study: In this study we aimed to assess the impact of thrombocytopenia on ACS patients.

Patients and Methods: We collected data of 608 ACS patients who presented to our hospital during the year 2017. Patients were divided into 2 groups according to the presence of initial thrombocytopenia: Group 1 with initial thrombocytopenia $(n=123)$ and group 2 without initial thrombocytopenia $(n=485)$. Platelet count less than or equal $150.000 / \mathrm{ml}$ was used to define thrombocytopenia. Endpoints were in-hospital and 6 months Major Adverse Cardiovascular and Cerebrovascular Events (MACCE).

Results: Group 1 patients were significantly older and, more likely with a history of acute coronary syndrome $(38.2 \%$ versus $26.7 \%, p=0.049)$, and more likely to present as non ST elevation myocardial infarction $(39 \%$ versus $26.7 \%$, $p=0.037)$. In hospital outcome showed significantly higher risk of cumulative MACCE in group 1 patients (12.2\% versus $2.3 \%, p=0.002$ ) and a similar result was found after at least 6 months follow-up $(19.3 \%$ versus $9.6 \%, p=0.041)$. Both groups had similar incidence of bleeding and heart failure. Group 1 patients had significantly higher incidence of in hospital MACCE $(p=0.038)$, but this was not significant after 6 months follow-up.
\end{abstract}

Conclusions: ACS patients with mild thrombocytopenia had similar bleeding risk in comparison to patients with normal count during the hospital stay and after 6 months of followup, higher risk of in hospital MACCE and so they should be managed as patients with normal platelet counts.

Key Words: Acute coronary syndromes - Thrombocytopenia - Percutaneous coronary intervention.

Correspondence to: Dr. Sherif W. Ayad, The Department of Cardiology, Faculty of Medicine, Alexandria University, Egypt

\section{Introduction}

THE incidence of Acute Coronary Syndromes (ACS) either ST-Segment Elevation Myocardial Infarction (STEMI) or Non ST-Segment Elevation Myocardial Infarction (NSTEMI) is increasing globally and it become most common cause of death worldwide. According to the latest guidelines, primary Percutaneous Coronary Intervention (PCI) in STEMI or early PCI in high-risk NSTEMI patients combined with antithrombotic drugs are the treatment of choice [1-3]

Diagnosis and classification of thrombocytopenia is based on the platelet count. The current classification of thrombocytopenia is mild (platelet count 100,000 to $150,000 / \mathrm{microL})$, moderate $(50,000$ to $99,000 / \mathrm{microL})$, or severe $(<50,000 /$ microL) $[4,5]$

\footnotetext{
List of Abbreviations:

ACS : Acute Coronary Syndromes.

PCI : Percutaneous Coronary Intervention.

MACCE : Major Adverse Cardiovascular and Cerebrovascular Events.

STEMI : ST Segment Elevation Myocardial Infarction.

NSTEMI : None ST Segment Elevation Myocardial Infarction.

EF : Ejection Fraction.

MR : Mitral Regurge.

DAPT : Dual Antiplatelet therapy.

$\mathrm{Hb} \quad$ : Haemoglobin.

LM : Left Main.

LAD : Left Anterior Descending.

LCX : Left Circumflex.

RCA : Right Coronary Artery.

LR : Low Risk.

HR : High Risk.

IR : Intermediate Risk.

VLR : Very Low Risk.

VHR : Very High Risk.

GP IIb/IIIa inhibitor : Glycoprotein IIb/IIIa inhibitor.
} 
There are several pathological conditions leading to thrombocytopenia such as decreased platelet production, increased platelet consumption or platelet sequestration [6-8].

The incidence of thrombocytopenia in general is approximately $13 \%$, while the incidence in ACS patients is about $5 \%$. Previous trials have shown that elderly, patients with diabetes, liver impairment, renal diseases, heart failure or previous cardiovascular diseases had the highest incidence [9]. The presence of baseline initial thrombocytopenia in ACS patients is a significant predictor of worse outcomes. In a study conducted by Yadav et al., they concluded that the presence of thrombocytopenia at baseline was an independent predictor of mortality at 1 year with no association with major or minor bleeding rates at 30 days [10]

The exact mechanisms beyond the correlation between thrombocytopenia and increased risk of ischemic events are still not clear. Some studies suggested that thrombocytopenia may be a marker of heavy burden of atherosclerosis predisposing to increased platelet consumption or reflecting increased thrombosis [11,12].

Recent guidelines recommend the use of Dual Antiplatelet Therapy (DAPT) in management of ACS patients. Currently there is no clear recommendation regarding the duration or type of DAPT used in this cohort of patients. ACS patients with thrombocytopenia are challenging in their management due to their high bleeding and ischeamic risks [13].

Also this group of patients have been excluded or underrepresented in major DAPT in ACS trials as TRITON-TIMI 38, PLATO, CURE and CHAMPION PHONIX trials $[\mathbf{9 , 1 4}]$

In this study we aimed to assess the impact of initial thrombocytopenia on short and midterm outcomes of patients with ACS managed with PCI.

\section{Patients and Methods}

\section{Study design:}

This is a retrospective observational study conducted on consecutive ACS patients who presented to the Alexandria Main University Hospital, Alexandria, Egypt between 1 January and 31 December 2017. The inclusion criteria were established diagnosis ACS fulfilling guidelines recommendation for PCI treatment [3]. The exclusion criteria were previous $\mathrm{CABG}$, cardiogenic shock, previous PCI of the same culprit vessel and severe Left Main (LM) coronary artery disease. The study population included 608 patients who were classified into two groups:

- Group 1: Included 123 ACS patients with initial thrombocytopenia platelet count less than $150 \mathrm{X}$ $10^{3} /$ microliter treated with PCI.

- Group 2: Included 485 ACS patients with initial normal platelet count more than $150 \times 10^{3}$ / microliter treated with PCI.

An informed consent was obtained from every patient or the legal guardians. The study was approved by the Local Ethics Committee.

\section{Data collection:}

All patients' demographic data were collected including age, gender, comorbidities (hypertension, diabetes, prior ACS, dyslipidemia), obtained PCI procedure details including access site, the culprit artery, number of diseased vessels, the use of antithrombotic treatment (acetyl salicylic acid, clopidogrel, ticagrelor, heparin, enoxaparin, and glycoprotein IIb/IIIa inhibitors). Also, any subsequent procedure related complications e.g., heart failure, stroke, or bleeding were documented. From laboratory data, we registered haemoglobin, platelet count, creatinine and Troponin I levels on admission and peak levels during the hospital stay. From echocardiography the following information were collected; Ejection Fraction (EF) and Mitral Regurgitation (MR). Baseline CRUSADE bleeding risk score and GRACE risk score were also calculated $[15,16]$

\section{Clinical endpoint measurements:}

The endpoints of the study were Major Adverse Cardiac and Cerebrovascular Events (MACCE) during hospital stay and at 6 months follow-up. MACCE was defined as death, re-infarction, need for revascularization, heart failure, stroke, and bleeding.

\section{Statistical analysis:}

Data were analyzed using the Statistical Package for Social Sciences (SPSS version 20.0. Armonk, NY: IBM Corp) [17]. We described qualitative data using number and percent and we described quantitative data using range (minimum and maximum), mean, standard deviation and median. Significance of the obtained results was judged at the 5\% level. The used tests were Chi-square test for categorical variables to compare between different groups, Fisher's Exact or Monte Carlo correction for chisquare when more than $20 \%$ of the cells have expected count less than 5 , student $t$-test for normally distributed quantitative variables, to compare between two studied groups, Mann Whitney test 
for abnormally distributed quantitative variables, to compare between two studied groups and regression to detect the most independent/affecting factor for MACCE.

\section{Results}

Patients characteristics: Both patient groups (1 and 2) were well matched with respect to demographic data and clinical characteristics except for the age, history of previous ACS, and diagnosis of NSTEMI at presentation which were significantly higher in group 1. The baseline characteristics of both groups are presented in (Table 1).

Table (1): Baseline characteristics of the studied populations.

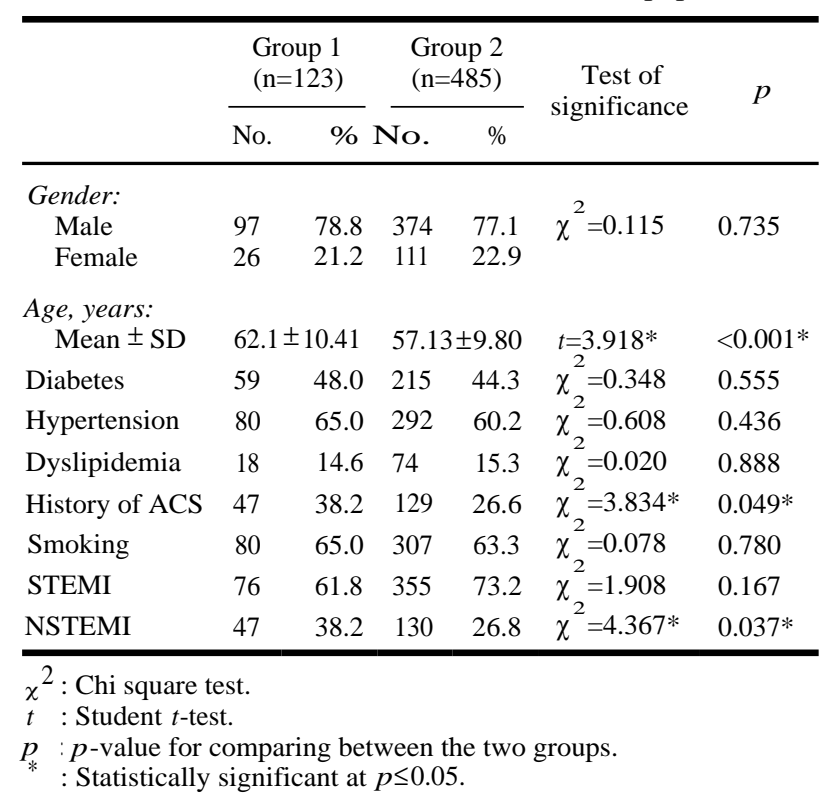

Table (2): Laboratory and echo cardiographic data of the studied population.

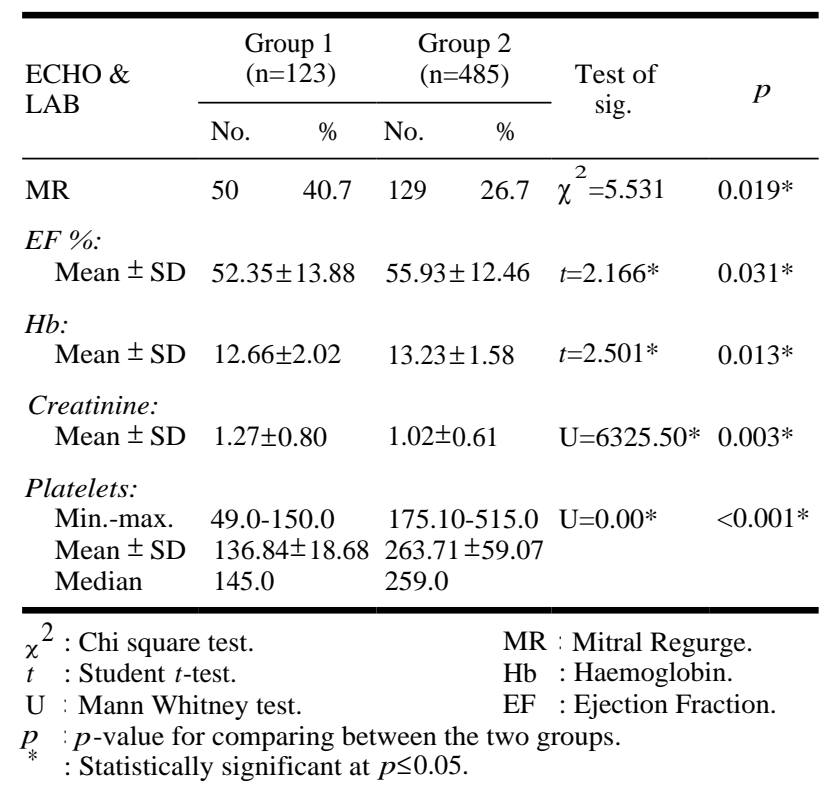

Echocardiography and laboratory results: Thrombocytopenic patients had significantly higher incidence of MR and lower EF. The prevalence of anemia was significantly higher in group 1 than in group $2(12.66 \pm 2.02$ vs. $13.23 \pm 1.58, p=0.013)$, serum creatinine levels were also higher in group 1 than in group 2 . The incidence of thrombocytopenia in our study was $20.2 \%, 117$ patients had mild thrombocytopenia, five patients had moderate thrombocytopenia and one patient had severe thrombocytopenia. The baseline echocardiography and laboratory results of both groups are presented in (Table 2).

Crusade bleeding risk score and Grace risk score $[15,16]$ : Patients in group 1 had signicantly higher Crusade bleeding and Grace risk scores than patients in group 2 as shown in (Table 3 ).

Table (3): Crusade bleeding and Grace risk scores of the studied population.

\begin{tabular}{|c|c|c|c|c|c|c|}
\hline & \multicolumn{2}{|c|}{$\begin{array}{l}\text { Group 1 } \\
(n=123)\end{array}$} & \multicolumn{2}{|c|}{$\begin{array}{l}\text { Group } 2 \\
(n=485)\end{array}$} & \multirow{2}{*}{$\begin{array}{l}\text { Test } \\
\text { of sig. }\end{array}$} & \multirow{2}{*}{$p$} \\
\hline & No. & $\%$ & No. & $\%$ & & \\
\hline $\begin{array}{l}\text { - Crusade: } \\
\text { Risk: } \\
\text { VLR } \\
\text { VHR } \\
\text { LR } \\
\text { MR } \\
\text { HR }\end{array}$ & $\begin{array}{l}17 \\
25 \\
33 \\
25 \\
23\end{array}$ & $\begin{array}{l}13.8 \\
20.3 \\
26.8 \\
20.3 \\
18.7\end{array}$ & $\begin{array}{l}196 \\
37 \\
159 \\
52 \\
41\end{array}$ & $\begin{array}{l}40.50 \\
7.6 \\
32.8 \\
10.7 \\
8.4\end{array}$ & $\begin{array}{l}\chi^{2}= \\
33.378\end{array}$ & $<0.001 *$ \\
\hline $\begin{array}{l}\text { Score: } \\
\quad \text { Min.-max. } \\
\text { Mean } \pm \text { SD. } \\
\text { Median }\end{array}$ & \multicolumn{2}{|c|}{$\begin{array}{l}7.0-76.0 \\
35.72 \pm 15.95 \\
36.0\end{array}$} & \multicolumn{2}{|c|}{$\begin{array}{l}8.0-76.0 \\
26.08 \pm 14.06 \\
23.0\end{array}$} & $\begin{array}{l}\mathrm{U}= \\
5027.50 *\end{array}$ & $<0.001 *$ \\
\hline $\begin{array}{l}\text { - Grace: } \\
\text { Risk: } \\
\text { LR } \\
\text { HR } \\
\text { IR }\end{array}$ & $\begin{array}{l}42 \\
29 \\
52\end{array}$ & $\begin{array}{l}34.1 \\
23.6 \\
42.3\end{array}$ & $\begin{array}{l}247 \\
56 \\
182\end{array}$ & $\begin{array}{l}51.1 \\
11.5 \\
37.4\end{array}$ & $\begin{array}{l}\chi^{2}= \\
10.036\end{array}$ & $0.007 *$ \\
\hline $\begin{array}{l}\text { Score: } \\
\quad \text { Min.-max. } \\
\text { Mean } \pm \text { SD } \\
\text { Median }\end{array}$ & $\begin{array}{l}50.0 \\
101 \\
102\end{array}$ & 23.89 & $\begin{array}{l}33.0 \\
89.3 \\
89.0\end{array}$ & $\begin{array}{l}177.0 \\
\pm 26.63\end{array}$ & $\begin{array}{l}t= \\
3.802 *\end{array}$ & $<0.001^{*}$ \\
\hline $\begin{array}{l}\text { In hospital } \\
\text { mortality: } \\
\text { Min.-max. } \\
\text { Mean } \pm \text { SD } \\
\text { Median }\end{array}$ & $\begin{array}{l}0.20 \\
2.5 \\
1.70\end{array}$ & & $\begin{array}{l}0.10 \\
2.29 \\
1.10\end{array}$ & $\begin{array}{l}32.50 \\
=4.07\end{array}$ & $\begin{array}{l}\mathrm{U}= \\
6401.0 *\end{array}$ & $0.005^{*}$ \\
\hline $\begin{array}{l}\text { Death from } \\
\text { admission- } \\
6 \text { months: } \\
\text { Min.-max. } \\
\text { Mean } \pm \text { SD } \\
\text { Median }\end{array}$ & $\begin{array}{l}0.70 \\
5.48 \\
4.0\end{array}$ & & $\begin{array}{l}0.40 \\
4.26 \\
3.0\end{array}$ & $\begin{array}{l}40.0 \\
-5.79\end{array}$ & $\begin{array}{l}\mathrm{U}= \\
5918.50 *\end{array}$ & $<0.001^{*}$ \\
\hline $\begin{array}{l}\text { After } 1 \text { year: } \\
\text { Min.-max. } \\
\text { Mean } \pm \text { SD } \\
\text { Median }\end{array}$ & $\begin{array}{l}0.80 \\
7.70 \\
5.50\end{array}$ & & $\begin{array}{l}0.50 \\
5.42 \\
3.0\end{array}$ & $\begin{array}{l}63.0 \\
=8.04\end{array}$ & $\begin{array}{l}U= \\
4996.50 *\end{array}$ & $<0.001 *$ \\
\hline $\begin{array}{ll}\text { LR } & : \text { Low Ris } \\
\text { VLR }: \text { Very Lo } \\
\text { MR } & \text { Moderat } \\
\text { HR } & \text { : High Ri } \\
t & : \text { Student } \\
p_{*} & : p \text {-value } \\
& : \text { Statistic }\end{array}$ & $\begin{array}{l}\text { W Ris } \\
\text { Risk } \\
\text { k. } \\
\text { t-test. } \\
\text { or co }\end{array}$ & $g$ & $\begin{array}{l}\mathrm{VH} \\
\mathrm{IR} \\
\chi^{2} \\
\mathrm{U}\end{array}$ & $\begin{array}{l}\text { : Very } \\
\text { Interm } \\
: \text { Chi } \\
: \text { Man } \\
\text { the two }\end{array}$ & $\begin{array}{l}\text { High Risk. } \\
\text { diate Risk. } \\
\text { quare test. } \\
\text { Whitney test } \\
\text { groups. }\end{array}$ & \\
\hline
\end{tabular}


Procedural characteristics of the studied population: With regard to the PCI data, the prevalence of multivessel disease was not different between the two groups, as was the culprit artery and the access site either femoral or radial. Also, the antiplatelet treatment with clopidogrel, ticagrelor or GP IIb/IIIa inhibitors did not differ. None of the patients in the two groups received fibrinolytic therapy. All patients in the two groups received Drug Eluting Stents (DES) and no patient had procedure related dissection or perforation. All patients received in-hospital medical treatment and follow-up according to the latest ACS guidelines [3]. Data of the procedural characteristics of the studied population are summarized in (Table 4).

Table (4): Procedural characteristics of the studied population.

\begin{tabular}{|c|c|c|c|c|c|}
\hline \multirow{2}{*}{ PCI details } & \multicolumn{2}{|c|}{$\begin{array}{l}\text { Group 1 } \\
(n=123)\end{array}$} & \multicolumn{2}{|c|}{$\begin{array}{l}\text { Group } 2 \\
(n=485)\end{array}$} & \multirow{2}{*}{$p$} \\
\hline & No. & $\%$ & No. & $\%$ & \\
\hline \multicolumn{6}{|l|}{ Access site: } \\
\hline Radial & 12 & 9.8 & 33 & 6.8 & \multirow[t]{2}{*}{0.404} \\
\hline Femoral & 111 & 90.2 & 452 & 93.2 & \\
\hline LM & 7 & 5.7 & 111 & 2.3 & 0.205 \\
\hline LAD & 78 & 63.4 & 285 & 58.8 & 0.449 \\
\hline $\mathrm{RCA}$ & 54 & 43.9 & 177 & 36.6 & 0.238 \\
\hline $\mathrm{LCx}$ & 50 & 40.7 & 229 & 47.3 & 0.284 \\
\hline Ramus & 5 & 4.1 & 4 & 0.8 & 0.110 \\
\hline \multicolumn{6}{|l|}{ Antiplatelets: } \\
\hline Clopidogrel & 75 & 60.9 & 278 & 57.3 & 0.410 \\
\hline Ticagrelor & 48 & 39.1 & 207 & 42.7 & 0.425 \\
\hline GP IIb/IIIa inhibitor & 2 & 1.6 & 16 & 3.3 & 0.345 \\
\hline
\end{tabular}

$p \quad: p$-value for comparing between the two groups

Statistically significant at $p \leq 0.05$.

LM : Left Main.

RCA : Right Coronary Artery.

LAD : Left Anterior Descending.

LCX : Left Circumflex.

\section{In hospital outcomes:}

In hospital cumulative MACCE was higher in group 1 (12.2\% vs. $2.3 \%)$ compared to group 2 , the difference between the two was significant ( $p$ $=0.002$ ). Five patients in group 1 and seven patients in group 2 died, mostly because of arrhythmia (ventricular fibrillation) and the rest developed intractable cardiogenic shock and pulmonary edema. The need for revascularization, incidence of heart failure, reinfarction or bleeding was not different between groups. Although there was statistically high ischemic stroke prevalence in group 1, we are unable to ascertain an exact explanation for it. One possible practice-based explanation for this finding is that in group 1 we used more thrombus aspiration catheters during PCI than in group 2. Hemorrhagic stroke or major bleeding did not occur in any patient of the 2 groups. The data of in-hospital outcomes are summarized in (Table 5).
Table (5): In hospital outcomes of the studied population.

\begin{tabular}{|c|c|c|c|c|c|c|c|}
\hline \multirow{2}{*}{\multicolumn{2}{|c|}{$\begin{array}{l}\text { Hospital } \\
\text { outcomes }\end{array}$}} & \multicolumn{2}{|c|}{$\begin{array}{l}\text { Group } 1 \\
(\mathrm{n}=123)\end{array}$} & \multicolumn{2}{|c|}{$\begin{array}{l}\text { Group } 2 \\
(\mathrm{n}=485)\end{array}$} & \multirow[t]{2}{*}{$x^{2}$} & \multirow[t]{2}{*}{$p$} \\
\hline & & No. & $\%$ & No. & $\%$ & & \\
\hline \multicolumn{2}{|l|}{ MACCE } & 15 & 12.2 & 11 & 2.3 & $9.452^{*}$ & $0.002 *$ \\
\hline \multicolumn{2}{|l|}{ Death } & 5 & 4.1 & 7 & 1.5 & 1.525 & $\mathrm{FE} p=0.269$ \\
\hline \multicolumn{8}{|c|}{ Revascularization: } \\
\hline \multirow{2}{*}{\multicolumn{2}{|c|}{$\begin{array}{l}\text { Non TVR } \\
\text { TVR }\end{array}$}} & 2 & 1.6 & 0 & 0.0 & 2.147 & \multirow{2}{*}{$\begin{array}{l}\mathrm{FE}_{p=0.234} \\
\mathrm{FE}_{p=0.612}\end{array}$} \\
\hline & & 2 & 1.6 & 4 & 0.8 & 0.404 & \\
\hline \multicolumn{2}{|l|}{ MI } & 4 & 3.3 & 4 & 0.8 & 2.036 & $\mathrm{FE} p=0.201$ \\
\hline \multirow{2}{*}{\multicolumn{2}{|c|}{$\begin{array}{l}\text { CVS: } \\
\quad \text { Ischem }\end{array}$}} & & & & & & \multirow{3}{*}{$\mathrm{FE} p=0.026^{*}$} \\
\hline & & 5 & 4.1 & 0 & 0.0 & $5.432^{*}$ & \\
\hline Hemor & rrhagic & 0 & 0.0 & 0 & 0.0 & & \\
\hline \multicolumn{2}{|l|}{$\mathrm{HF}$} & 30 & 24.4 & 74 & 15.3 & 3.340 & 0.068 \\
\hline \multirow{2}{*}{\multicolumn{8}{|c|}{$x_{\mathrm{E}}^{2} \quad:$ Chi square test. }} \\
\hline & \multirow{2}{*}{\multicolumn{7}{|c|}{$\begin{array}{l}\text { Fisher Exact. } \\
\text { Major Adverse Cardiovascular and Cerebrovascular Events. }\end{array}$}} \\
\hline $\begin{array}{l}\text { MACCE } \\
\text { TVR }\end{array}$ & & & & & & & \\
\hline $\begin{array}{l}\text { TVR } \\
\text { MI }\end{array}$ & \multirow{2}{*}{\multicolumn{7}{|c|}{$\begin{array}{l}\text { : Major Adverse Cardiovascular and Cerebrovascular Events. } \\
\text { : Target Vessel Revascularization. }\end{array}$}} \\
\hline $\begin{array}{l}\text { MI } \\
\text { CVS }\end{array}$ & \multicolumn{5}{|c|}{$\begin{array}{l}\text { Myocardial Infarction. } \\
\text { : Cerebrovascular Stroke. }\end{array}$} & & \\
\hline $\begin{array}{l}\mathrm{CVS} \\
\mathrm{HF}\end{array}$ & \multicolumn{7}{|c|}{$\begin{array}{l}\text { : Cerebrovascular Stroke } \\
\text { : Heart Failure. }\end{array}$} \\
\hline & \multicolumn{7}{|c|}{$\begin{array}{l}p \text {-value for comparing between the two groups. } \\
: \text { Statistically significant at } p \leq 0.05 \text {. }\end{array}$} \\
\hline
\end{tabular}

6 months follow-up: Fourteen patients from group 1 and seventeen patients from group 2 were lost during follow-up. The composite MACCE was higher in group 1 (19.3 vs. 9.6\%, $p=0.041)$ as was the incidence of cardiac death (6.4 vs. $0.9 \%)$ compared to group 2, the difference between the two was significant $(p=0.032)$. The need for revascularization, re-infarction, the incidence of heart failure, bleeding or stroke was not different between groups. The data of 6 months follow-up outcomes are summarized in (Table 6).

Table (6): 6 months follow-up outcomes of the studied population.

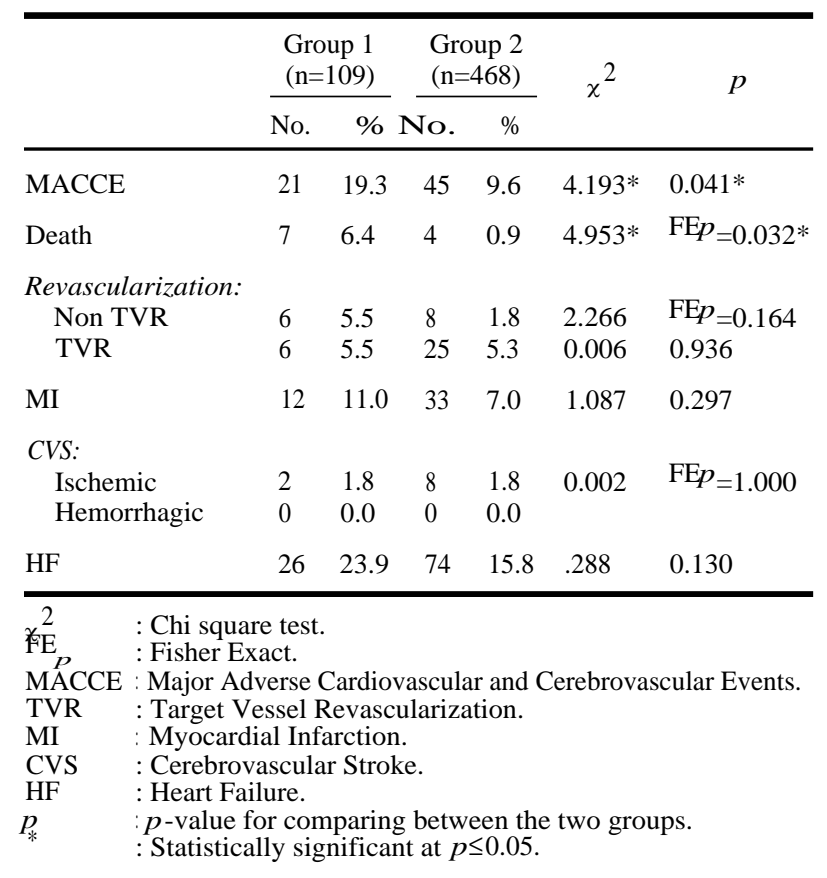


Relationship between initial thrombocytopenia and MACCE: Multivariable regression modeling showed that mild thrombocytopenia contributed to higher incidence of in hospital MACCE $(p=0.038)$.
However, it did not show any significant correlation with MACCE after 6 months followup. Tables $(7,8)$ summarize multivariable regression modeling in hospital and after 6 months follow-up.

Table (7): Univariate and multivariate analysis for the parameters affecting in hospital MACCE $(n=608)$ for total sample.

\begin{tabular}{|c|c|c|c|c|}
\hline \multirow{2}{*}{$\begin{array}{l}\text { MACCE } \\
\text { (Hospital outcomes) }\end{array}$} & \multicolumn{2}{|c|}{ Univariate } & \multicolumn{2}{|c|}{ \#Multivariate } \\
\hline & $p$ & OR $(95 \%$ C.I $)$ & $p$ & OR $(95 \%$ C.I $)$ \\
\hline Females & 0.082 & $2.429(0.895-6.591)$ & & \\
\hline Age (years) & 0.201 & $1.030(0.984-1.078)$ & & \\
\hline $\begin{array}{l}\text { Past history: } \\
\text { Diabetes } \\
\text { Hypertension } \\
\text { Dyslipidemia } \\
\text { ACS } \\
\text { Smoking }\end{array}$ & $\begin{array}{l}0.886 \\
0.892 \\
0.271 \\
0.035^{*} \\
0.779\end{array}$ & $\begin{array}{l}0.932(0.355-2.455) \\
0.934(0.349-2.499) \\
0.316(0.041-2.450) \\
2.874 *(1.079-7.51) \\
0.868(0.325-2.324)\end{array}$ & 0.588 & $1.457(0.413-5.137)$ \\
\hline STEMI & 0.600 & $0.736(0.234-2.317)$ & & \\
\hline Non STEMI & 0.951 & $1.032(0.373-2.855)$ & & \\
\hline $\mathrm{EF} \%$ & $0.001 *$ & $0.942 *(0.910-0.976)$ & 0.144 & $0.962(0.913-1.013)$ \\
\hline $\mathrm{Hb}$ & $0.005 *$ & $0.679 *(0.517-0.892)$ & 0.060 & $4.301(0.940-19.678)$ \\
\hline Platelets & $0.005^{*}$ & $0.985 *(0.975-0.995)$ & $0.038 *$ & $0.988(0.977-0.99)$ \\
\hline Creatinine & $0.012 *$ & $1.702 *(1.123-2.579)$ & 0.196 & $1.425(0.833-2.436)$ \\
\hline Troponin & 0.339 & $1.012(0.987-1.037)$ & & \\
\hline Score (Crusade) & $0.001 *$ & $1.052 *(1.021-1.083)$ & 0.676 & $0.988(0.934-1.045)$ \\
\hline Score (Grace) & $0.023 *$ & $1.021 *(1.003-1.039)$ & 0.807 & $1.005(0.965-1.047)$ \\
\hline In hospital mortality & 0.108 & $1.072(0.985-1.167)$ & & \\
\hline 6 Months mortality & 0.060 & $1.056(0.998-1.119)$ & 0.770 & $0.986(0.898-1.083)$ \\
\hline $\begin{array}{l}\text { Antiplatelets: } \\
\text { Clopidogrel } \\
\text { Ticagrelor } \\
\text { GP IIbIIIa inhibitors }\end{array}$ & $\begin{array}{l}0.952 \\
0.708 \\
0.028 *\end{array}$ & $\begin{array}{l}0.965(0.304-3.058) \\
0.783(0.218-2.816) \\
7.250 *(1.233-42.6)\end{array}$ & 0.192 & $5.874(0.411-83.990)$ \\
\hline PCI details & & & & \\
\hline $\begin{array}{l}\text { Access site: } \\
\text { Radial } \\
\text { Femoral }\end{array}$ & $\begin{array}{l}0.667 \\
0.667\end{array}$ & $\begin{array}{l}0.635(0.080-5.025) \\
0.635(0.080-5.025)\end{array}$ & & \\
\hline LM & $0.001 *$ & $10.952 *(2.768-43.33)$ & 0.078 & $4.913(0.836-28.861)$ \\
\hline LAD & 0.317 & $1.721(0.594-4.987)$ & & \\
\hline RCA & 0.380 & $1.53(0.589-4.016)$ & & \\
\hline $\mathrm{LCx}$ & 0.314 & $1.64(0.626-4.309)$ & & \\
\hline Ramus & 0.999 & - & & \\
\hline Death & 0.999 & - & & \\
\hline Non TVR & 0.999 & - & & \\
\hline TVR & 0.999 & - & & \\
\hline MI & 0.999 & - & & \\
\hline Ischemic & 0.999 & - & & \\
\hline Hemorrhagic & 0.999 & - & & \\
\hline
\end{tabular}

\footnotetext{
OR : Odd's ratio.

C.I : Confidence Interval.

\# $\quad$ All variables with $p<0.05$ was included in the multivariate.

* : Statistically significant at $p \leq 0.05$.

ACS : Acute Coronary Syndromes.

PCI : Percutaneous Coronary Intervention.

MACCE : Major Adverse Cardiovascular and Cerebrovascular Events.

STEMI : ST Segment Elevation Myocardial Infarction.

NSTEMI : None ST Segment Elevation Myocardial Infarction.

EF : Ejection Fraction.

MR : Mitral Regurge

DAPT : Dual Antiplatelet Therapy.

$\mathrm{Hb}$ : Haemoglobin

LM : Left Main.

LAD : Left Anterior Descending.

LCX : Left Circumflex.

RCA : Right Coronary Artery.

GP IIb/IIIa inhibitor : Glycoprotein IIb/IIIa inhibitor.
} 
Table (8): Univariate and multivariate analysis for the parameters affecting 6 months at least followup MACCE $(n=577)$ for total sample.

\begin{tabular}{|c|c|c|c|c|}
\hline \multirow{2}{*}{$\begin{array}{l}\text { MACCE } \\
\text { (Follow-up) }\end{array}$} & \multicolumn{2}{|c|}{ Univariate } & \multicolumn{2}{|c|}{ \#Multivariate } \\
\hline & $p$ & OR $(95 \%$ C.I $)$ & $p$ & OR (95\% C.I) \\
\hline Females & 0.155 & $0.450(0.150-1.351)$ & & \\
\hline Age (years) & 0.055 & $1.036(0.99-1.075)$ & & \\
\hline $\begin{array}{l}\text { Past history: } \\
\text { Diabetes } \\
\text { Hypertension } \\
\text { Dyslipidemia } \\
\text { ACS } \\
\text { Smoking }\end{array}$ & $\begin{array}{l}0.338 \\
0.562 \\
0.698 \\
0.002 * \\
0.938\end{array}$ & $\begin{array}{l}1.444(0.681-3.059) \\
1.262(0.576-2.766) \\
1.230(0.433-3.489) \\
3.262 *(1.516-7.018) \\
1.032(0.469-2.268)\end{array}$ & 0.116 & $3.117(0.756-12.846)$ \\
\hline STEMI & 0.265 & $0.585(0.228-1.502)$ & & \\
\hline Non STEMI & 0.155 & $1.740(0.811-3.732)$ & & \\
\hline $\mathrm{EF} \%$ & 0.257 & $0.983(0.955-1.012)$ & & \\
\hline $\mathrm{Hb}$ & 0.233 & $0.881(0.715-1.085)$ & & \\
\hline Platelet count & 0.104 & $0.995(0.990-1.001)$ & & \\
\hline Troponin & 0.321 & $1.010(0.990-1.031)$ & & \\
\hline Score (Crusade) & $0.042 *$ & $1.024 *(1.001-1.047)$ & 0.298 & $0.983(0.951-1.016)$ \\
\hline Score (Grace) & $0.029 *$ & $1.016 *(1.002-1.030)$ & 0.770 & $0.995(0.965-1.026)$ \\
\hline In hospital mortality & 0.143 & $1.060(0.981-1.145)$ & & \\
\hline 6 Months mortality & $0.020^{*}$ & $1.061 *(1.009-1.115)$ & 0.226 & $1.076(0.956-1.212)$ \\
\hline $\begin{array}{l}\text { Antiplatelets: } \\
\text { Clopidogrel } \\
\text { Ticagrelor } \\
\text { GP IIbIIIa inhibitors }\end{array}$ & $\begin{array}{l}0.155 \\
0.227 \\
0.201\end{array}$ & $\begin{array}{l}2.221(0.740-6.664) \\
0.507(0.168-1.526) \\
3.117(0.547-17.767)\end{array}$ & & \\
\hline PCI details & & & & \\
\hline $\begin{array}{l}\text { Access site: } \\
\text { Radial } \\
\text { Femoral }\end{array}$ & $\begin{array}{l}0.263 \\
0.263\end{array}$ & $\begin{array}{l}0.310(0.040-2.407) \\
3.225(0.415-25.046)\end{array}$ & & \\
\hline $\mathrm{LM}$ & $0.010 *$ & $6.679 *(1.580-28.236)$ & $0.047 *$ & $5.213 *(1.020-26.630)$ \\
\hline LAD & 0.060 & $2.257(0.965-5.280)$ & & \\
\hline RCA & 0.386 & $1.395(0.657-2.962)$ & & \\
\hline $\mathrm{LCx}$ & 0.285 & $1.507(0.711-3.193)$ & & \\
\hline Ramus & 0.999 & - & & \\
\hline Death & 0.999 & - & & \\
\hline Non TVR & 1.000 & - & & \\
\hline TVR & 0.999 & & & \\
\hline MI & 0.999 & & & \\
\hline Ischemic & 0.999 & & & \\
\hline Hemorrhagic & 0.999 & - & & \\
\hline
\end{tabular}

OR : Odd's ratio.

C.I : Confidence Interval.

\# : : All variables with $p<0.05$ was included in the multivariate.

* : : Statistically significant at $p \leq 0.05$.

ACS : Acute Coronary Syndromes.

PCI : Percutaneous Coronary Intervention.

MACCE : Major Adverse Cardiovascular and Cerebrovascular Events.

STEMI : ST Segment Elevation Myocardial Infarction.

NSTEMI : None ST Segment Elevation Myocardial Infarction.

EF : Ejection Fraction.

MR : Mitral Regurge.

DAPT : Dual Antiplatelet Therapy.

$\mathrm{Hb} \quad$ : Haemoglobin.

LM : Left Main.

LAD : Left Anterior Descending.

LCX : Left Circumflex.

RCA : Right Coronary Artery.

GP IIb/IIIa inhibitor : Glycoprotein IIb/IIIa inhibitor. 


\section{Discussion}

Baseline initial thrombocytopenia defined as platelet count less than or equal $150,000 / \mathrm{ml}$ is not an uncommon finding among patients presenting with ACS and previous trials showed its correlation with worse outcome $[\mathbf{1 0 , 1 8 , 1 9 ]}$. The objective of this study was to evaluate the impact of initial thrombocytopenia on the outcomes of ACS patients treated with PCI either in-hospital or after 6 months follows-up. Our results show that the incidence of thrombocytopenia in ACS patients is approximately $20.2 \%$. This finding is comparable to the results of Sinkovic A et al., who reported $21.3 \%$ incidence of thrombocytopenia among ACS patients [22]. Other studies conducted by McClure et al., [11], Yeh et al., [20] Eikelboom JW [21], and Yadav et al., [10] reported lower incidence ranging from 1$7 \%$. In our study thrombocytopenic patients were significantly older, with history of previous ACS, and more likely to present as NSTEMI. These results are consistent with previous studies. Yadav et al., showed that baseline thrombocytopenia was more common in older men with diabetes mellitus, anemia, previous MI, and renal insufficiency and was associated with higher rates of long-term adverse ischemic events after PCI [10].

Our findings can be summarized in showing significant increase in cumulative MACCE while in-hospital and after 6 months follow in thrombocytopenic patients. This finding is compatible with other studies. Yadav et al., and Sinkovic A et al., $[10,22]$ also reported higher incidence of in hospital mortality in thrombocytopenic patients. Despite that, the prevalence of developed heart failure, reinfarction need for revsaculariztion and bleeding was not different from non thrombocytopenic patients.

Univariate and multivariate logistic regression analyses were performed to identify the factors associated with MACCE either during hospitalization or after six months follow-up. This showed that baseline thrombocytopenia was an independent predictor of in-hospital MACCE $(p=0.038)$, but this was not the same after six months follow-up. These findings were similar to results reported by Yadavetal and Sinkovic A et al., [10,22] that showed association between thrombocytopenia and adverse outcomes.

Major bleeding did not occur in any patient of the two groups neither in hospital or after 6 months follow-up due to the fact that most of our patients had only mild thrombocytopenia only 6 patients had platelet counts below 100,000/ml.

\section{Limitations:}

This study has some obvious limitations. First most of the patients had mild thrombocytopenia, which may have affected the results. Second this was a single center trial including relatively small number of patients. We still need more trials including larger number of patients and including ACS patients with moderate or severe thrombocytopenia.

\section{Conclusion:}

Our study shows that ACS patients with mild thrombocytopenia had significantly higher MAACE in comparison to non thrombocytopenic patients either in-hospital or after 6 months of follow-up, but similar bleeding risk and so they should be managed as patients with normal platelet counts.

\section{Author contributions:}

Conceptualization, S.A., M.S., and O.N.; Methodology, S.A., M.S., and M.S.; Software, R.S.; Validation, S.A.; Formal analysis, S.A., M.S., and O.N.; Investigation, S.A., M.S., and M.S.; Writingoriginal draft preparation, S.A.; Writing-review and editing, S.A., M.S., and O.N. All authors have read and agreed to the published version of the manuscript.

Funding: This research received no external funding.

\section{Acknowledgements: None.}

Institutional Review Board Statement: The study was conducted according to the guidelines of the Declaration of Helsinki, and approved by Faculty of Medicine, Alexandria University Ethics Committee.

Informed Consent Statement: Informed consent was obtained from all subjects involved in the study.

Data Availability Statement: Data available in a publicly accessible repository.

Conflicts of Interest: The authors declare no conflict of interest.

\section{References}

1- TURPIE A.G.: Burden of disease: Medical and economic impact of acute coronary syndromes. Am. J. Manag. Care, 12 (16 Suppl): S430-4, 2006.

2- MEADOWS E., P. BAE J., ZAGAR A., SUGIHARA T., RAMASWAMY K., McCRACKEN R., et al.: Rehospitalization following percutaneous coronary intervention for commercially insured patients with acute coronary syndrome: A retrospective analysis. BMC Res. Notes, 5: $342,2012$. 
3- BRAUNWALD E., ANTMAN E.M., BEASLEY J.W., CALIFF R.M., CHEITLIN M.D., HOCHMAN J.S., et al.: ACC/AHA guideline update for the management of patients with unstable angina and non-ST-segment elevation myocardial infarction--2002: Summary article: A report of the American College of Cardiology/American Heart Association Task Force on Practice Guidelines (Committee on the Management of Patients With Unstable Angina). Circulation, 106 (14): 1893-900, 2002.

4- GAUER R.L. and BRAUN M.M.: Thrombocytopenia. Am. Fam. Physician, 85 (6): 612-22, 2012.

5- ITO S., WATANABE H., MORIMOTO T., YOSHIKAWA Y., SHIOMI H., SHIZUTA S., et al.: Impact of Baseline Thrombocytopenia on Bleeding and Mortality After Percutaneous Coronary Intervention. Am. J. Cardiol., 121 (11): 1304-14, 2018.

6- IZAK M. and BUSSEL J.B.: Management of thrombocytopenia. F1000Prime Reports, 6: 45, 2014

7- PATEL P.D., SAMANICH J.M., MITCHELL W.B. and MANWANI D.: A unique presentation of Wiskott-Aldrich syndrome in relation to platelet size. Pediatr. Blood Cancer, 56 (7): 1127-9, 2011.

8- ASTER R.H.: Immune thrombocytopenia caused by glycoprotein IIb/IIIa inhibitors. Chest, 127 (2 Suppl): 53s-9s, 2005.

9- McCARTHY C.P., STEG G. and BHATT D.L.: The management of antiplatelet therapy in acute coronary syndrome patients with thrombocytopenia: A clinical conundrum. Eur. Heart J., 38 (47): 3488-92, 2017.

10- YADAV M., GENEREUX P., GIUSTINO G., MADHAVAN M.V., BRENER S.J., MINTZ G., et al.: Effect of Baseline Thrombocytopenia on Ischemic Outcomes in Patients With Acute Coronary Syndromes Who Undergo Percutaneous Coronary Intervention. Can. J. Cardiol., 32 (2): 226-33, 2016.

11- McCLURE M.W., BERKOWITZ S.D., SPARAPANI R., TUTTLE R., KLEIMAN N.S., BERDAN L.G., et al.: Clinical significance of thrombocytopenia during a nonST-elevation acute coronary syndrome. The platelet glycoprotein IIb/IIIa in unstable angina: Receptor suppression using integrilin therapy (PURSUIT) trial experience. Circulation, 99 (22): 2892-900, 1999.

12- RAPHAEL C.E., SPOON D.B., BELL M.R., PSALTIS P.J., KIDD S., LOH S.X., et al.: Effect of Preprocedural Thrombocytopenia on Prognosis After Percutaneous Coronary Intervention. Mayo Clin. Proc., 91 (8): 1035-44, 2016.

13- LEVINE G.N., BATES E.R., BITTL J.A., BRINDIS R.G., FIHN S.D., FLEISHER L.A., et al.: ACC/AHA Guideline Focused Update on Duration of Dual Antiplatelet Therapy in Patients With Coronary Artery Disease: A Report of the American College of Cardiology/American Heart Association Task Force on Clinical Practice Guidelines. J. Am. Coll. Cardiol., 68 (10): 1082-115, 2016.

14- WIVIOTT S.D., BRAUNWALD E., McCABE C.H., MONTALESCOT G., RUZYLLO W., GOTTLIEB S., et al.: Prasugrel versus clopidogrel in patients with acute coronary syndromes. N. Engl. J. Med., 357 (20): 2001 $15,2007$.

15- ALVAREZ-ALVAREZ B., RAPOSEIRAS-ROUBIN S., ABU-ASSI E., CAMBEIRO-GONZALEZ C., GESTALROMANI S., LOPEZ-LOPEZ A., et al.: Is 6-month GRACE risk score a useful tool to predict stroke after an acute coronary syndrome? Open Heart, 1 (1): e000123, 2014.

16- SUBHERWAL S., BACH R.G., CHEN A.Y., GAGE B.F., RAO S.V., NEWBY L.K., et al.: Baseline risk of major bleeding in non-ST-segment-elevation myocardial infarction: The CRUSADE (Can Rapid risk stratification of Unstable angina patients Suppress ADverse outcomes with Early implementation of the ACC/AHA Guidelines) Bleeding Score. Circulation, 119 (14): 1873-82, 2009.

17- KIRKPATRICK L.A. and FEENEY B.C.: A simple guide to IBM SPSS statistics for version 20.0. Student ed. Belmont, Calif.: Wadsworth, Cengage Learning; 2013.

18- SANCHIS-GOMAR F., PEREZ-QUILIS C., LEISCHIK R. and LUCIA A.: Epidemiology of coronary heart disease and acute coronary syndrome. Ann. Transl. Med., 4 (13): 256,2016

19- CAIXETA A., DANGAS G.D., MEHRAN R., FEIT F., NIKOLSKY E., LANSKY A.J., et al.: Incidence and clinical consequences of acquired thrombocytopenia after antithrombotic therapies in patients with acute coronary syndromes: Results from the Acute Catheterization and Urgent Intervention Triage Strategy (ACUITY) trial. Am. Heart J., 161 (2): 298-306.e1, 2011.

20- YEH R.W., WIVIOTT S.D., GIUGLIANO R.P., MORROW D.A., SHUI A., QIN J., et al.: Effect of thrombocytopenia on outcomes following treatment with either enoxaparin or unfractionated heparin in patients presenting with acute coronary syndromes. Am. J. Cardiol., 100 (12): 1734-8, 2007.

21- EIKELBOOM J.W., ANAND S.S., MEHTA S.R., WEITZ J.I., YI C. and YUSUF S.: Prognostic significance of thrombocytopenia during hirudin and heparin therapy in acute coronary syndrome without ST elevation: Organization to Assess Strategies for Ischemic Syndromes (OASIS-2) study. Circulation, 103 (5): 643-50, 2001.

22- SINKOVIC A. and MAJAL M.: The Impact of Thrombocytopenia on Outcome in Patients with Acute Coronary Syndromes: A Single Center Retrospective Study. Biomed. Res. Int., 2015: 907304, 2015. 


\section{تآثير ثقص الصفائح الدموية الآولى على النتائج قصيرة ومتوسطة الآمل



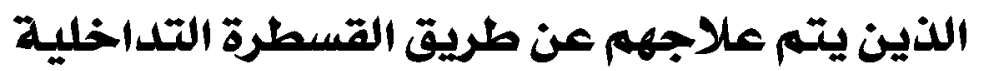

الفرض من البحث: تقييم تآثير نقص الصفائح الدموية الآولى على النتائج قصيرة ومتوسطة الآمد فى مرضى متلازمة قصود الشرايين التاجية الذين يتم علاجهم عن طريق القسطرة التداخية.

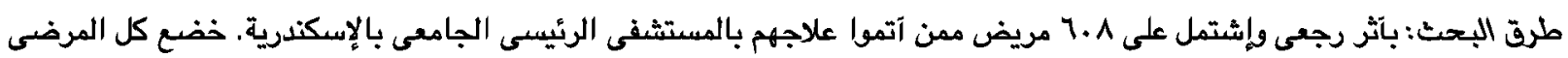

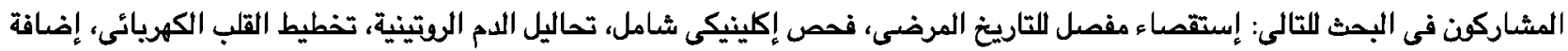

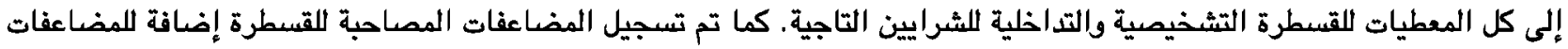

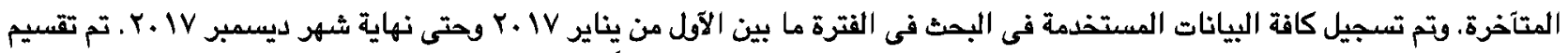

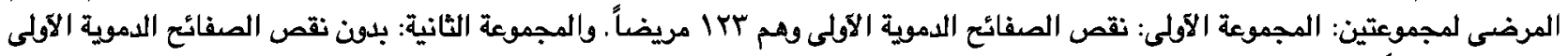

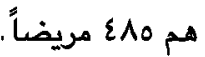

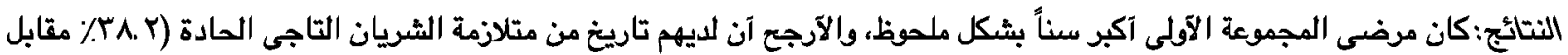

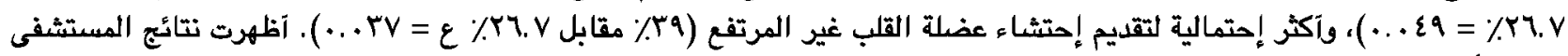

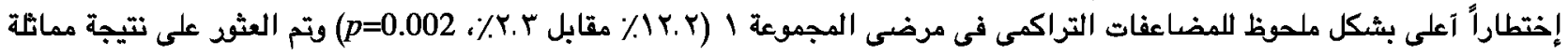

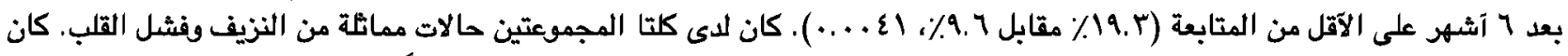

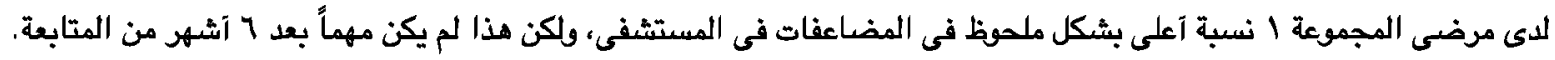

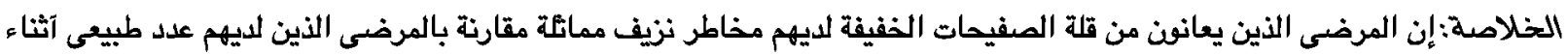

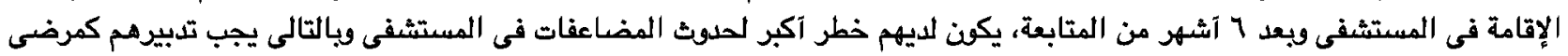

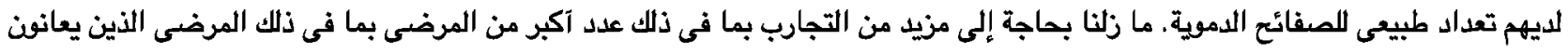
من ثلة الصفيحات المعتلة آو الشديدة. 